\title{
EL APRENDIZAJE CON VIDEOJUEGOS. EXPERIENCIAS Y BUENAS PRÁCTICAS REALIZADAS EN LAS AULAS ESPAÑOLAS
}

\section{Álvaro Pérez García}

\section{RESUMEN}

Desde su nacimiento, los nativos digitales conviven con los smartphones, las tablets, los ordenadores portátiles o los videojuegos, siendo habitual que desde los primeros años de vida se interesen por su funcionamiento y aprendan a manejarlos correctamente desde edades muy tempranas. A pesar del espectacular auge de la industria de los videojuegos, los sistemas educativos siguen resistiéndose de forma generalizada a utilizar sistemáticamente estas plataformas de ocio interactivo en los desarrollos curriculares, haciendo oídos sordos a sus enormes posibilidades creativas y didácticas. Una posible causa de tal situación es la escasa formación del profesorado en las competencias básicas necesarias para el uso eficaz de los videojuegos en las aulas.

En este trabajo de investigación se ha realizado una revisión bibliográfica acerca de las distintas investigaciones y experiencias sobre la utilización de los videojuegos en las aulas españolas para situarnos en la realidad actual de su implementación.

Palabras clave: videojuegos, TIC, competencia digital, revisión bibliográfica.

\section{TITLE: LEARNING WITH VIDEOGAMES, EXPERIENCES AND GOOD PRACTICE CARRIED OUT IN SPANISH CLASSROOMS}

\section{ABSTRACT}

Since they were born, digital natives coexist with smartphones, tablets, laptops or videogames, and it is considered normal that from the first years of life they take an interest in how these work and they learn how to operate them from early childhood. In spite of the spectacular boom in the videogame industry, the educational systems in general, still resist to systemically use these platforms of interactive leisure in curricular development, ignoring their enormous creative and teaching possibilities.

A possible reason for this situation is the insufficient training of teachers in the basic competences needed for the efficient use of videogames in the classroom. In this work, we have carried out a bibliographical revision of the different experiences and research on the use of videogames in Spanish classrooms in order to situate the present day reality of their implementation.

Keywords: videogames, ICT, digital competence, bibliographical revision.

Correspondencia con el autor: Álvaro Pérez García. < alvaroperez@fundacionsafa.es. Centro de Profesorado SAFA (Adscrito a Universidad de Jaén). Original recibido: 28-II-13. Original aceptado: 25-03-14 


\section{Los videojuegos y su caracterización}

Los videojuegos, en tanto dispositivo electrónico y elemento activo de la industria del entretenimiento, "son el medio audiovisual en el que se está produciendo todo el proceso de la integralidad propuesto por el encuentro entre la informática y la televisión, y que han contribuido a prefigurar la nueva generación de la cultura de la comunicación" (Levis, 1997).

Marquès Graells (2000) entiende por videojuegos todo tipo de juego digital interactivo, con independencia de su soporte (ROM interno, cartucho, disco magnético u óptico, on-line) y plataforma tecnológica (máquina de bolsillo, videoconsola conectable al TV, teléfono móvil, máquina recreativa, microordenador, ordenador de mano, vídeo interactivo).

Igualmente Ortega (2002) señala, que "se trata de narraciones audiovisuales de naturaleza digital que se presentan en forma de aventura gráfica, simulación o arcade, y representan una alternativa a los tradicionales relatos cinematográficos o televisivos".

También Maldonado (1999) concibe los videojuegos como instrumentos lúdicos que requieren de un soporte electrónico, es decir de una plataforma de juego electrónica (consola doméstica, PC, máquinas recreativas, etc.).

Aguiar y Farray (2003) exponen que los videojuegos "surgen en la década de los cuarenta, concretamente en 1946 con la aparición de la computadora electrónica (ENIAC) que es la base de los juegos electrónicos. El Gobierno de EE.UU., una vez terminada la Segunda Guerra Mundial, abrió laboratorios-museos para que el público contemplara los avances científicos. En uno de estos museos, el científico Willy Higinbotham pensó en hacer un juego que se pudiera ver en la pantalla de televisión para que la gente que acudiese al museo se entretuviese. Este primer juego era muy sencillo para dos personas, en donde una pelotita rebotaba en la pantalla y los jugadores debían golpearla con la raqueta".

La historia de los videojuegos ha sido recopilada por Estallo (1995) quien destaca que el primer videojuego nace en los años 1940, siendo un simulador de vuelo para el entrenamiento de pilotos, creado por técnicos americanos.

Para este autor, hasta 1962 no surgió la tercera generación de videojuegos, que reduce su tamaño y coste. En 1969 nació el microprocesador, donde se podía almacenar mayor cantidad de información en un espacio mucho más reducido, convirtiéndose en la base fundamental de ordenadores, videojuegos y calculadoras. 
En la primera década de los años 70 nació el disco flexible y posteriormente, en 1972 el primer juego, llamado PONG, que consistía en una rudimentaria partida de tenis o ping-pong. En 1977, la empresa ATARI lanzó al mercado el primer sistema de videojuegos en cartucho, que alcanzó un gran éxito en Estados Unidos y provocó, al mismo tiempo, una primera preocupación sobre los posibles efectos de los videojuegos en la conducta de los niños.

Tras una rápida evolución, en la que el constante aumento de la potencia de los microprocesadores y de la memoria permitió nuevas mejoras, en I986, NINTENDO lanzó su primer sistema de videojuegos que permitió la presentación de unos juegos impensables nueve años atrás. La calidad del movimiento, el color y el sonido, la imaginación de los creadores de juegos y el considerable abaratamiento relativo de los mismos a comienzos de los 90 , provocaron que en nuestro país se extendieran de manera masiva los videojuegos creados por las dos principales compañías, SEGA y NINTENDO, pasando en poco tiempo a constituirse en uno de los juguetes preferidos por los niños.

La extensión masiva de los videojuegos en los años 90 provocó una segunda oleada de investigaciones, desde la medicina, la sociología, la psicología y la educación, además de la preocupación y las valoraciones que dichos juegos han recibido por parte de padres, educadores y principalmente los medios de comunicación, para quienes generalmente los videojuegos son vistos como algo negativo y perjudicial. Las más prestigiosas universidades, revistas y publicaciones están haciendo un hueco a la preocupación por uno de los temas preferidos a la hora de elegir los juegos, no solo de los niños y adolescentes, sino también de jóvenes y adultos.

En este sentido Balaguer (2002) señala que los videojuegos han dejado de ser una mera forma de entretenimiento para pasar a una forma de expresión cultural de finales del siglo $X X$.

Pascual y Ortega (2007, pp. 208-209) caracterizan los videojuegos subrayando:

I) El poder de atracción y generación de interés que impulsan las máquinas y las tecnologías en las nuevas generaciones.

2) La posibilidad de interactuar con la máquina. A diferencia de la televisión, los videojuegos nos proponen ser protagonistas de primer orden en el progreso y avance de lo que ocurre, reclama del usuario la plena concentración para el éxito y de ello es plenamente consciente. 
3) Se puede jugar sólo o contra otro y se puede utilizar, gracias a Internet y a las consolas de bolsillo o los teléfonos móviles, desde cualquier lugar. En la actualidad se reconocen tres formas diferentes de difusión: unilateral, reciprocidad y multiplicidad.

4) La calidad de los diseños mediante la enorme carga gráfica, recreando entornos fantásticos, de gran realismo y acción. Se caracterizan por su dinamismo. Integran una diversidad de notaciones simbólicas adecuadamente integradas: informaciones textuales, sonido, música, animación, vídeo, fotografías, imágenes en tres dimensiones, lo cual nos hace intuir el desarrollo de una percepción de las formas, el color. La música y el movimiento diferentes. Aunque por el momento las investigaciones sobre los medios todavía no han llegado a ninguna conclusión relevante sobre la eficacia real de la combinación de las diferentes notaciones simbólicas en un solo medio.

5) Algunos autores hablan de la originalidad y la creatividad de los guiones, pero tal originalidad es falsamente otorgada, pues en los últimos años asistimos a una reproducción de las películas de cine que conectan con el público infantil y juvenil, pues lo que se busca es asegurar el éxito comercial en primer lugar.

6) Se busca el juego inmediato con instrucciones orales.

7) Generan una cultura propia convirtiéndolos en un tema de intercambio y relación a partir de los símbolos, personajes, lenguaje, gestos... que generan.

También, Huerta Rojas (2005, p. I 76) destaca que los videojuegos "se caracterizan principalmente porque son juegos de competencia y combate, basados y contenidos del poder de dominio, la violencia y el sexismo, que son jugados principalmente por hombres adolescentes y jóvenes. Mediante el ritual del juego, los adolescentes aprenden a conocerse a si mismos en la medida en que corroboran o no el cumplimiento adecuado del deber, desear y poder ser hombres jóvenes, contenido en el desiderátum cultural, a partir de lo cual conocen y reconocen sus capacidades, habilidades y destrezas de jugador virtual". Esta caracterización permite avanzar hacia la taxonomización de los videojuegos. 


\section{Clasificación de los videojuegos}

Clasificar videojuegos es tarea ardua y compleja. Ciertos autores los clasifican según sus tipologías, otros los organizan de forma similar a los géneros cinematográficos y algunos se aventuran a sistematizarlos por criterios pedagógicos. Levis (1997) subraya la dificultad de clasificar de forma exhaustiva y precisa los videojuegos por géneros, ya que aunque hay muchos juegos que poseen sus características propias, la gran mayoría conforman productos híbridos que mezclan ingredientes de diferentes géneros.

Así, Estallo (1995) cataloga los videojuegos en modalidades según sus características:

\begin{tabular}{c|l|l}
\hline \multicolumn{1}{c|}{ Tipo de juego } & \multicolumn{1}{|c}{ Características } & \multicolumn{1}{c}{ Modalidades } \\
\hline \multirow{2}{*}{ ARCADE } & $\begin{array}{l}\text { Ritmo rápido de juego } \\
\text { Tiempo de reacción mínimo } \\
\text { Atención focalizada } \\
\text { Componente estratégico secundario }\end{array}$ & $\begin{array}{l}\text { Plataformas } \\
\text { Laberintos } \\
\text { Deportivos } \\
\text { Dispara y olvida }\end{array}$ \\
\hline \multirow{2}{*}{ SIMULADORES } & $\begin{array}{l}\text { Baja influencia del tiempo de reacción } \\
\text { Estrategias complejas y cambiantes }\end{array}$ & $\begin{array}{l}\text { Instrumentales } \\
\text { Situacionales } \\
\text { Deportivos }\end{array}$ \\
\hline ESTRATEGIA & $\begin{array}{l}\text { Se adopta una identidad específica } \\
\text { Solo se conoce el objetivo final del juego } \\
\text { Desarrollo mediante órdenes y objetos }\end{array}$ & $\begin{array}{l}\text { Aventuras gráficas } \\
\text { Juegos de rol }\end{array}$ \\
\hline JUEGOS DE MESA & Juegos de Guerra \\
\hline
\end{tabular}

Tabla I. Clasificación de los videojuegos (Estallo, 1995) 
Por su parte, Marquès Graells (2000) realiza la siguiente clasificación según su tipología:

\begin{tabular}{lr}
\hline \multicolumn{2}{c}{ CLASIFICACIÓN DE LOS VIDEOJUEGOS } \\
\hline TIPO DE VIDEOJUEGO & CONSIDERACIONES \\
\hline
\end{tabular}

- Pueden contribuir al desarrollo psicomotor y de la orientación espacial de los

Arcade (juegos tipo plataforma, luchas...) estudiantes, aspecto especialmente útil en el caso de los más pequeños.

EJEMPLOS: Pacman, Mario, - Riesgos a considerar: nerviosismo, estrés y hasta angustia que pueden Sonic, Doom, Quake, Street Fighter, Arcanoid. manifestar algunos alumnos ante las dificultades que encuentran para controlar a los personajes del juego.

- Conviene limitar el tiempo que se dedique a esta actividad y observar los comportamientos de los pequeños para ayudarles y detectar posibles síntomas de estar sometidos a una tensión excesiva.

\begin{tabular}{|c|c|}
\hline $\begin{array}{l}\text { Deportes } \\
\text { EJEMPLOS: FIFA, PC Futbol, } \\
\text { NBA, Formula I GrandPrix, } \\
\text { Need For Speed. }\end{array}$ & $\begin{array}{l}\text { - Permiten la ejercitación de diversas habilidades de coordinación } \\
\text { psicomotora y profundizar en el conocimiento de las reglas y estrategias de } \\
\text { los deportes. } \\
\text { - En algunos casos también se pueden alcanzar niveles altos de estrés. }\end{array}$ \\
\hline $\begin{array}{l}\text { Juegos de aventura y rol } \\
\text { EJEMPLOS: King Quest, } \\
\text { Indiana Jones, Monkey Island, } \\
\text { Final Fantasy, Tomb Raider, } \\
\text { Pokémon, Ultima Online. }\end{array}$ & $\begin{array}{l}\text { - Pueden proporcionar información y constituir una fuente de motivación } \\
\text { hacia determinadas temáticas que luego se estudiarán de manera más } \\
\text { sistemática en clase. } \\
\text { - Una de las preocupaciones de los educadores deberá ser promover la } \\
\text { reflexión sobre los valores y contravalores que se consideran en el juego. }\end{array}$ \\
\hline $\begin{array}{l}\text { Simuladores y } \\
\text { constructores (aviones, } \\
\text { maquinarias, ciudades...) } \\
\text { EJEMPLO: Simulador de vuelo } \\
\text { Microsoft, Sim City, Tamagotchi, } \\
\text { The Incredible Machine, Theme } \\
\text { Park }\end{array}$ & $\begin{array}{l}\text { - Permiten experimentar e investigar el funcionamiento de máquinas, } \\
\text { fenómenos y situaciones. } \\
\text { - Además de controlar posibles estados de tensión excesiva en algunos } \\
\text { alumnos, conviene advertir a los estudiantes que están ante un modelo } \\
\text { (representación simplificada de la realidad - a veces presentan una realidad } \\
\text { imaginaria-) y que por lo tanto en el mejor de los casos sólo constituyen una } \\
\text { aproximación a los fenómenos que se dan en el mundo físico. } \\
\text { - La realidad siempre es mucho más compleja que las representaciones de los } \\
\text { mejores simuladores. }\end{array}$ \\
\hline $\begin{array}{l}\text { Juegos de estrategia } \\
\text { EJEMPLOS: Estratego, Warcraft, } \\
\text { Age of Empires, Civilitation, } \\
\text { Lemmings, Black \& White, } \\
\text { Centurion }\end{array}$ & $\begin{array}{l}\text { - Exigen administrar unos recursos escasos (tiempo, dinero, vidas, armas...) } \\
\text { prever los comportamientos de los rivales y trazar estrategias de actuación } \\
\text { para lograr unos objetivos. } \\
\text { - Quizá los mayores peligros de estos juegos sean de carácter moral, por los } \\
\text { contravalores que muchas veces asumen y promueven. Resulta conveniente } \\
\text { organizar actividades participativas que permitan analizar y comentar estos } \\
\text { aspectos con los jugadores. }\end{array}$ \\
\hline $\begin{array}{l}\text { Puzzles y juegos de lógica } \\
\text { EJEMPLO: } 7^{\text {th }} \text { Guest, Tetris }\end{array}$ & $\begin{array}{l}\text { - Desarrollan la percepción espacial, la lógica, la imaginación y la creatividad. } \\
\text { - No contemplamos riesgos específicos para este tipo de juegos, aunque como } \\
\text { pasa con todos los videojuegos conviene evitar una excesiva adicción que } \\
\text { podría conducir a un cierto aislamiento y falta de ejercicio físico. }\end{array}$ \\
\hline $\begin{array}{l}\text { Juegos de preguntas } \\
\text { EJEMPLO: Trivial, Carmen } \\
\text { Sandiego }\end{array}$ & $\begin{array}{l}\text { - Los juegos de preguntas pueden servir para repasar determinados } \\
\text { conocimientos de todo tipo }\end{array}$ \\
\hline
\end{tabular}

Tabla 2. Clasificación de los videojuegos según su tipología (Marquès Graells, 2000) 
También Pascual y Ortega (2007, pp. 2II-2I2) agrupan los videojuegos atendiendo a los diferentes géneros existentes:

- Acción: el jugador interactúa constantemente con diversidad de elementos.

- Carreras: el jugador puede controlar y hacer circular su vehículo por circuitos que reproducen localidades y paisajes, distintos tipos de asfalto, condiciones climáticas o situación que alteran el modo de conducción, circuitos de carreras reales u otros ficticios con obstáculos...

- Estrategia: existen varios subgéneros, como juegos de estrategia bélica, estrategia deportiva o juegos de estrategia en tiempo real. El jugador pone en práctica su capacidad de análisis y de gestión de la información, la toma de decisiones y la gestión de recursos para conseguir unos objetivos.

- Aventura: centran la acción sobre un argumento o historia de ficción. El jugador asume el rol de uno o varios de los personajes.

- Deporte: emulan entornos deportivos reales, ciñéndose a las normas de cada deporte y en algunos casos simulando los entornos y los jugadores reales de cada temporada. Los niveles de simulación permiten elegir jugadores, crear equipos, seleccionar materiales y campos de juego, organizar o participar en torneos, ligas...

- Simulación: ponen a disposición de los jugadores un escenario para construir y desarrollar la acción del juego, como por ejemplo una ciudad, una civilización...

Una clasificación similar es la realizada por Begoña Gros y el Grup F9 (2004) cuando hablan de:

- Juegos de acción (llamados también arcades), son los que se empezaron a desarrollar para las videoconsolas y suelen depender más de la coordinación entre ojos y manos que de la trama del juego (Tekken, Mortal Combat).

- Juegos de estrategia que hacen hincapié en el pensamiento y la planificación lógica (Age of Empires).

- Juegos de aventura, suelen llevar al jugador a un viaje de exploración y resolución de problemas (Indiana Jones). 
- Juegos de rol, se parecen a los de aventura pero, en vez de basarse en la resolución de enigmas, dependen de la evolución de los personajes. También se les llama MUD (Multi User Domain) porque pueden jugarse en red con varios usuarios al mismo tiempo (Final Fantasy VII).

- Juegos de deporte que simulan la estrategia básica de los deportes individuales o grupales (Golf, PC Futbol).

- Simuladores, que suelen recrear con todo detalle un objeto o proceso (SimCity 3000, Los Sims).

Teniendo esto en consideración, Huerta Rojas (2003, pp. 74-77) propone la siguiente clasificación:

- Juegos de lucha: consisten en una lucha cuerpo a cuerpo entre dos personajes elegidos y controlados por los jugadores. Estos enfrentan a diversos luchadores adiestrados en las artes marciales, y dependiendo del programa, poseen una serie de poderes que practican contra sus adversarios.

- Juegos de combate: son juegos cuyo único objetivo es disparar y matar todo aquello que aparezca en la pantalla, que por lo regular son hombres con características de delincuentes modernos, equipados con armamento sofisticado; en ocasiones, suelen ser acompañados por mujeres, quienes actúan como sus cómplices y por lo tanto reúnen características similares a las de ellos.

- Simuladores: videojuegos que tienen sus antecesores en los años 40 , cuando en la milicia se empezaron a emplear los simuladores para la instrucción en el manejo de aviones y carros de combate. En los juegos actuales, las y los participantes ponen a prueba sus habilidades de conductores para manejar vehículos -autos, motos y motos de agua, principalmente- a grandes velocidades, librando los rivales y peligros de las autopistas terrestres y marítimas.

- Juegos de deporte: estos juegos son de los que tienen mayor aceptación entre la población masculina. Se basan en algunos deportes profesionales que se juegan en muchas partes del mundo, como son el fútbol, el baloncesto, el béisbol, el jockey, el boxeo y el voley playa. Sus contenidos de competencia, lucha, triunfo, éxito y violencia se enmarcan en un supuesto ambiente deportivo. 
Dando un paso más, Farray, Aguiar y otros (2002) ofrecen la siguiente clasificación siguiendo criterios de utilidad educativa:

- Producciones cerradas: explícitamente violentas, en la que el único objetivo del juego es la destrucción del otro. Normalmente se apoya en valores sexistas, racistas de discriminación étnica y son una apología de la fuerza y la violencia (Mortal Kombat).

- Producciones medias: de contenido no violento pero de marcado carácter antidemocrático. Estos juegos representan una visión estereotipada de la realidad, ayudan a afrontar un pensamiento acrítico y alienante y refuerzan la adopción de contravalores (Theme Hospital).

- Producciones flexibles: videojuegos comerciales pero adaptables y apropiados para desarrollar contenidos curriculares (The Sims).

- Producciones educativas: material curricular, diseñado con propósito instructivo.

Gros (2009, pp. 15-16) nos habla de una nueva tipología, denominados videojuegos serios (serious games), que "tienen como objetivo usar las ventajas que proporcionan los videojuegos, pero cuyo objetivo fundamental no es el entretenimiento sino el aprendizaje".

Durall (2009, p. 19) profundiza más en esta tipología y añade que "los videojuegos serios tienen el objetivo de crear puentes entre lo lúdico y el mundo real y por este motivo han sido utilizados como medio para la educación, el aprendizaje de nuevos conceptos, la concienciación, la denuncia social o política e incluso la propaganda, la mercadotecnia y la publicidad. La apropiación de los videojuegos desde ámbitos tan diferentes se basa en la siguiente premisa: los videojuegos son un medio de comunicación más. Las temáticas de los Juegos Serios son muy diversas y abarcan cuestiones como la salud, el medio ambiente, la economía, los derechos humanos o la ciencia, entre muchas otras".

La normalización de criterios clasificatorios ha alcanzado cierta importancia desde que en 2003 se creara el sistema en Europa de etiquetaje de videojuegos, conocido por las siglas PEGI (Pan European Game Information). Su objetivo es ayudar a los progenitores europeos a tomar decisiones informadas a la hora de adquirir juegos de ordenador. Mediante un sistema pictogramas, se informa a los usuarios sobre el contenido de un determinado juego. Sustituyó a una serie de sistemas nacionales de clasificación por edades englobándolos en un único sistema que se utiliza ya en la 
mayor parte de Europa, en 30 países (Austria, Dinamarca, Hungría, Letonia, Noruega, Eslovenia, Bélgica, Estonia, Islandia, Lituania, Polonia, España, Bulgaria, Finlandia, Irlanda, Luxemburgo, Portugal, Suecia, Chipre, Francia, Israel, Malta, Rumanía, Suiza, República Checa, Grecia, Italia, Países Bajos, República Eslovaca y Reino Unido).

El sistema está respaldado por los principales fabricantes de consolas, incluidos SONY, Microsoft y NINTENDO, así como por editores y desarrolladores de juegos interactivos de toda Europa y fue desarrollado por la Federación de Software Interactivo de Europa (ISFE).

La clasificación PEGl por edad está destinada a garantizar que el contenido de los productos de entretenimiento (películas, los vídeos, los DVD y los juegos de ordenador), sea etiquetado por edades en función de su contenido. Éstas orientan pues a los consumidores (especialmente a los padres) y les ayudan a tomar la decisión sobre si deben comprar o no un producto concreto.

LaAsociación Española de Distribuidores y Editores de Software de Entretenimiento (ADESE) diseñó los logotipos que forman el sistema PEGI con una paleta de colores que informa sobre si un juego es apto para todos los públicos se basa en el lenguaje de los semáforos. Es decir, la tonalidad verde claro se asociará a edades de tres, siete años y superiores, mientras que la roja indicará que es adecuado sólo para adultos. En medio, el ámbar señala franjas comprendidas de entre doce y dieciséis años.

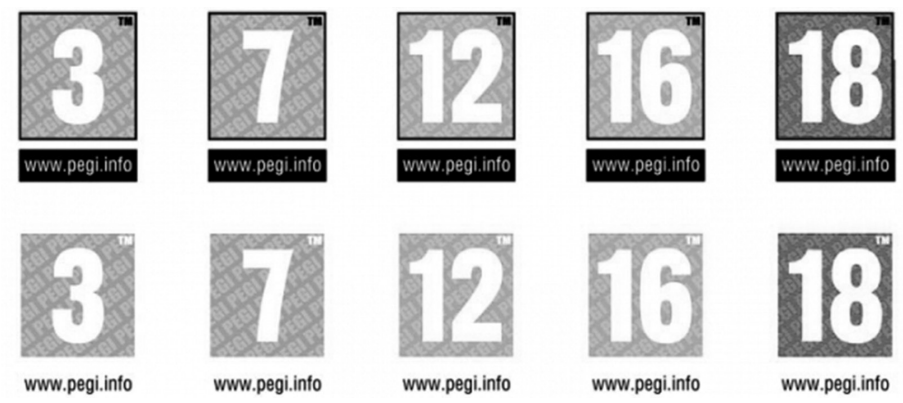

Imagen I. Logotipos de la clasificación por edades del sistema PEGI

Los sellos de edad se acompañarán de dibujos e iconos que muestran de forma gráfica la temática del juego. Por ejemplo, proporcionan datos acerca de si el contenido incluye lenguaje malsonante, actitudes racistas, contenidos sexuales o si provoca miedo. 


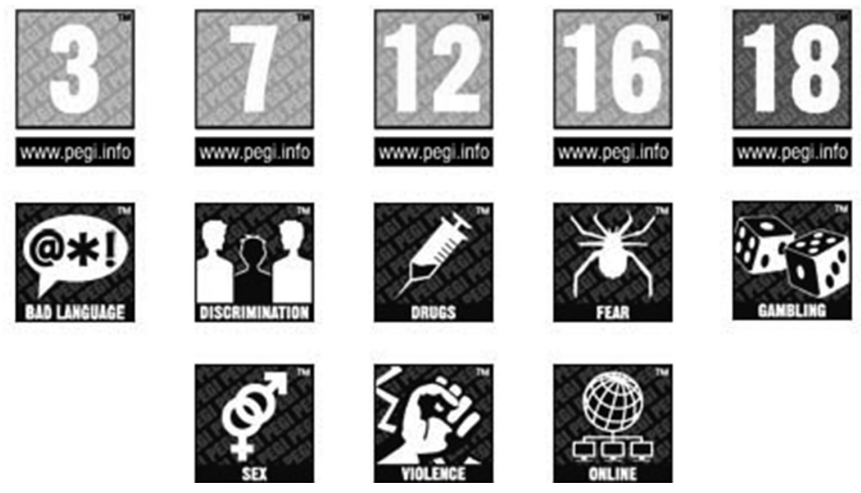

Imagen 2. Iconos del sistema PEGI para la catalogación por temáticas

Dichos iconos deberán aparecer en la caratula del videojuego, y los padres deberán entender estos símbolos en los siguientes términos:

- Violencia: el juego contiene representaciones violentas.

- Palabrotas o lenguaje soez: el juego contiene palabrotas.

- Miedo: el juego puede asustar o dar miedo.

- Sexo o desnudos: el juego contiene representaciones de desnudez y/o comportamientos sexuales o referencias sexuales.

- Drogas: el juego hace referencia o muestra el uso de drogas.

- Discriminación: el juego contiene representaciones o material que puede favorecer la discriminación.

Por otro lado, para optar a la etiqueta PEGI OK, el juego no puede contener ninguno de los elementos siguientes: violencia, actividad sexual o insinuación sexual, desnudo, lenguaje soez, juegos de apuestas, fomento o consumo de drogas, fomento del alcohol o tabaco, escenas de miedo.

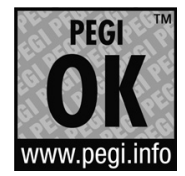

Imagen 3. Etiqueta PEGI OK 
ADESE ha credo un nuevo pictograma, PEGI Online, para catalogar la jugabilidad en Internet. Para que un videojuego lleve el distintivo PEGI Online, debe cumplir una serie de requisitos, entre otros, la obligación de mantener un sitio web libre de contenido ilegal u ofensivo, así como de enlaces que conduzcan a sitios dudosos, y se deberán añadir medidas que preserven la intimidad de los jóvenes cuando participen en juegos en línea. De lo que se trata es de prevenir uno de los problemas que plantea la red en relación con los menores, su vulnerabilidad ante ataques malintencionados.

Como dato relevante cabe citar que en nuestro país, en el año 2004 se creó en un entorno interuniversitario el "Observatorio del Videojuego" (http://www. observatoriovideojuegos.com/), que muestra un especial interés en el seguimiento del videojuego educativo y del infantil, y que en 2005 comenzó la ejecución de un estudio en el que se hizo un seguimiento pormenorizado a los diez géneros más representativos (arcade, acción, aventura gráfica, on-line, deportes, educativos, estrategia, carreras, infantiles y eróticos; Pérez Martín, 2008, p. 23).

\section{Videojuegos y aprendizaje}

La evolución visual de los videojuegos y su influencia en los usuarios es un interesantísimo ámbito de investigación poco desarrollado hasta el momento, si bien existe la certeza teórica de que la tridimensionalidad y la realidad virtual favorecen la intensidad de la "inmersión" perceptiva y emocional del jugador en el mundo virtual representado, equiparable posiblemente a la introducción de la perspectiva renacentista en la representación pictórica. Estas innovadoras vertientes tecnológico-creativas abren nuevas posibilidades a la creación de materiales didácticos hipermedia visualmente tridimensionales y dotados de sonido espacial (distribuido por cinco canales) especialmente útiles para alumnado con necesidades educativas especiales (Pascual y Ortega, 2007, p. 22I).

En este sentido, Marquès Graells (2000) afirma que los videojuegos representan un reto continuo para los usuarios que, además de observar y analizar el entorno, deben asimilar y retener información, realizar razonamientos inductivos y deductivos, construir y aplicar estrategias cognitivas de manera organizada y desarrollar determinadas habilidades psicomotrices (lateralidad, coordinación psicomotor...) para afrontar las situaciones problemáticas que se van sucediendo ante la pantalla. Aquí el jugador siempre se implica y se ve obligado a tomar decisiones y ejecutar acciones motoras continuamente, aspecto muy apreciado por los niños y jóvenes, generalmente con tendencia a la hiperactividad; en este sentido el juego supone un desahogo de tensiones. 
Garaigordobil (1990) plantea que cuando el niño juega, éste se desarrolla integralmente a nivel biopsicológico, físico y social. Igualmente, ha comprobado que cuando el niño juega con videojuegos desarrolla habilidades y destrezas propias de la psicología social que inciden en el proceso enseñanza-aprendizaje, de las cuales se mencionan las siguientes:

- Su capacidad para emplear símbolos aumenta, ya que por medio de estos juegos, puede representar diferentes fenómenos, analizar sus experiencias conscientes, planear, imaginar y actuar de manera previsora.

- El jugador, utiliza sus procesos de autorregulación de tal manera que puede controlar, seleccionar y organizar las influencias externas de modo que no se limita a reaccionar ante las situaciones lúdicas que tiene durante el juego.

- El sujeto interactúa con el entorno, de forma que durante el juego la persona maneja las riendas de la situación y establece los límites de su autonomía.

- La motivación y estimulación visual y auditiva de los videojuegos permite al jugador la resolución de diferentes niveles de problemas y dificultades, con lo cual se obtiene el dominio de habilidades y destrezas propias de la tecnología.

Para Gifford (199I) existen siete características que hacen de los videojuegos un medio de aprendizaje más atractivo y efectivo:

I. Permiten el ejercicio de la fantasía, sin limitaciones espaciales, temporales o de gravedad.

2. Facilitan el acceso a "otros mundos" y el intercambio de unos a otros a través de los gráficos, contrastando de manera evidente con las aulas convencionales y estáticas.

3. Favorecen la repetición instantánea y el intentarlo otra vez, en un ambiente sin peligro.

4. Permiten el dominio de habilidades. Aunque sea difícil, los niños pueden repetir las acciones, hasta llegar a dominarlas, adquiriendo sensación de control.

5. Facilitan la interacción con otros amigos, además de una manera no jerárquica, al contrario de lo que ocurre en el aula.

6. Hay una claridad de objetivos. Habitualmente, el niño no sabe qué es lo que está estudiando en matemáticas, ciencias o sociales, pero cuando juega al 
videojuego sabe que hay una tarea clara y concreta: abrir una puerta, rescatar a alguien, hallar un tesoro, etc. lo cual proporciona un alto nivel de motivación.

7. Favorece un aumento de la atención y del autocontrol, apoyando la noción de que cambiando el entorno, no el niño, se puede favorecer el éxito individual.

Etxeberría (1998) habla del éxito de los videojuegos desde el punto de vista psico-educativo, destacando las siguientes características:

- La influencia de la observación sobre los pensamientos, afectos y conductas.

- La capacidad humana de emplear símbolos permite representar los fenómenos, analizar su experiencia consciente, planear, imaginar y actuar de manera previsora.

- Los procesos de autorregulación juegan un papel central, seleccionando, organizando y filtrando las influencias externas.

- Hay una interacción continua entre el sujeto y el entorno.

Así, Pascual y Ortega (2007, p. 222) afirman que puede comprobarse que videojuegos electrónicos reúnen muchas de las características que exige la organización eficaz del aprendizaje social, especialmente su alto contenido en reforzadores que pueden agruparse en las siguientes categorías:

- Aquellos relativos al carácter lúdico de los aprendizajes.

- Los relacionados con la dificultad creciente y progresiva de adquirir ciertas habilidades.

- El ritmo individual de cada participante.

- El conocimiento claro de las tareas y los objetivos a conseguir.

- La posibilidad de repetir y corregir el ejercicio.

- La recompensa inmediata después de cada logro.

- El conocimiento de la existencia de un sistema determinado y definido de recompensas.

- El reconocimiento social de los logros adquiridos (compañeros,...).

- La posibilidad de inscribir los records o niveles máximos. 
- La constante superación del propio nivel.

- Los aplausos del público.

- La gratificación afectiva del beso de la chica o del chico, por ejemplo.

- La actividad participativa, manual, cardiovascular, etc.

- La identificación con héroes socialmente prestigiosos.

- La práctica de deportes o actividades socialmente valoradas.

- La estimulación visual, auditiva, kinestésica, actitudinal, etc.

En una obra reciente, Marín Díaz (2013) afirma que "la utilización de los videojuegos y juegos digitales como un recurso más en el desarrollo de un currículo inclusivo aporta una serie de beneficios educativos"

\section{Estudios y experiencias sobre el aprendizaje con videojuegos en España}

El objeto de este apartado es situar y reflexionar acerca de la utilización de los videojuegos como herramienta didáctica en las aulas españolas. Para ello se ha realizado una amplia revisión bibliográfica acerca de estas experiencias que comenzó por la búsqueda de revistas españolas situadas en las bases de datos Web of Science, SCOPUS y Scielo, sobre todo las situadas en las áreas de Comunicación y Ciencias Sociales. Posteriormente se realizó una nueva búsqueda de revistas y libros en la base de datos IN-RECS y en el repositorio DIALNET. Una vez localizada la información se procedió a la selección y gestión de la información, teniendo en cuenta los siguientes aspectos: antigüedad de la publicación, importancia de sus autores en el ámbito de estudio y novedad de la investigación o experiencia presentada. Por último, se procedió a la transformación de los resultados obtenidos en el texto que se muestra a continuación.

Sádaba y Naval (2008) realizaban una aproximación a la virtualidad educativa de los videojuegos, exponiendo que resulta necesario conocer las motivaciones que llevan a los jóvenes al uso de estas plataformas de ocio interactivo. En este sentido concluían que la necesidad del otro, la búsqueda común del entretenimiento, la necesaria cooperación con los otros para conseguir metas comunes en muchas plataformas, auguran al menos unas disposiciones que luego podrán ser reforzadas y empleadas en otros ámbitos. 
Conviene destacar también la propuesta metodológica de Torrico Franco (2008, pp. 27-30), basada en la utilización didáctica de la consola Nintendo DS. Ésta tiene sus antecedentes en la escuela japonesa Joshi Gakuen Junior High, que en junio de 2008 incorporó en sus clases de inglés la Nintendo DS, con el respaldo de la misma compañía de videojuegos para realizar una prueba piloto y comprobar así sus efectos educativos.

Morales (2009) concluye un estudio acerca del uso de los videojuegos como recurso de aprendizaje en educación afirmando que los videojuegos representan en la actualidad una de las herramientas más directas de los niños a la cultura informática. Los juegos informáticos poseen unos atributos propios y diferenciados de otros tipos de programas aunque buena parte del software educativo actual intenta seguir los diseños de los juegos para aumentar la motivación de los usuarios. No obstante, las diferencias en cuanto a formato de los juegos de ordenador y de los juegos educativos son todavía bastante evidentes.

Llorca (2009) estudió si las actitudes de los usuarios ante los videojuegos y la forma en que los utilizan repercuten significativamente en variables cognitivas, en concreto en la inteligencia espacial, la autoeficacia y el rendimiento académico, reconociendo la existencia de medidas de protección estructuradas que protegen al menor. Tras analizar una muestra formada por 266 participantes con edades comprendidas entre II y 16 años, junto con sus correspondientes progenitores, padres o madres, concluia que los videojuegos pueden representar "un vehículo pedagógico muy útil" para motivar la autoeficacia, una variable que mejora el rendimiento académico, y para ello veía necesario fomentar en padres, docentes y tutores el conocimiento de este tipo de ocio tecnológico como parte de la comunicación visual y de su utilización por parte de los menores para aprovechar su motivación en el ámbito de la enseñanza.

Estas conclusiones ponen de manifiesto, como señalan Pérez y Ortega (20I I), la necesidad de integrar la alfabetización audiovisual en la formación inicial de los maestros, otorgándole una especial importancia al uso, creación, gestión y evaluación de la utilización didáctica de los videojuegos.

Pérez García (2010) abría la posibilidad de utilizar algunas potencialidades de los videojuegos para fomentar la creatividad en el aula. En este sentido, afirma que la creación de cortometrajes digitales con la ayuda de software lúdico, es una herramienta de gran valor para superar la tecnofobia y favorecer la creatividad del profesorado en formación y del alumnado de los distintos niveles del sistema 
educativo. Para ello, realizó una experiencia con alumnado de tercero de magisterio de la Universidad de Granada en la que aprovechaba el interesante software del videojuego The Movies para utilizar de forma didáctica y creativa esta herramienta, y exponía la necesidad de integrar la alfabetización audiovisual en la formación inicial de los maestros, otorgándole una especial importancia al uso, creación, gestión y evaluación de la utilización didáctica de los videojuegos.

Por su parte, Armando (2010) realizó un estudio del videojuego World of Goo para mostrar, cómo un videojuego comercial nos puede orientar respecto de cuestiones a tener en cuenta en la producción de materiales educativos, sobre todo si pretendemos que los materiales propongan desafíos a nuestros alumnos debe dejar de ponerse el énfasis sólo en la producción de escenarios atractivos y vistosos para los estudiantes, ya que estos escenarios no provocarán desafíos en la medida en que no se vinculen internamente con los conocimientos a enseñar, orientando así los esfuerzos principales hacía al estudio didáctico profundo de esos conocimientos y de la manera en que son producidos para poder generar situaciones en las que los mismos sean necesarios.

En este mismo sentido, Zagalo (2010) realizó un estudio sobre el estado actual de la alfabetización mediática prestando especial atención al cine y los videojuegos, intentando analizar si algunos de los problemas encontrados a lo largo de la historia de la alfabetización cinematográfica han existido también en el proceso de la alfabetización en los videojuegos, e intentando unificar ambas visiones para captar la atención de las personas que trabajan en el campo de los videojuegos. Para ello, el estudio abarcó la alfabetización en los videojuegos en dos dimensiones, la dimensión cultural y la dimensión creativa. La dimensión cultural se definió a partir de los enfoques críticos y educacionales y la creativa mediante el diseño y la programación. Se concluía hablando de la necesidad de que exista un nivel de motivación en la alfabetización de los videojuegos, estimando urgente la implantación de una perspectiva de alfabetización basada en el diseño creativo y la programación y poniendo especial énfasis en el desarrollo del enfoque comunicativo.

La Asociación Española de Distribuidores y Editores de software de entretenimiento (ADESE, 20II) realizó un estudio donde no solo plantea el empleo de los videojuegos como ocio. Se plantean como auténticos canales de formación para el desarrollo de habilidades psicomotrices y prácticas (para cirujanos, mecánicos, pilotos, etc.) y para desarrollar habilidades y valores empresariales sobre toma de decisiones, transmisión de cultura de empresa. Aún van más allá cuando proponen el empleo de los videojuegos como terapia 
psicológica (en niños con déficit de atención, autismo, fobias, etc.) o físicas (para la rehabilitación motriz).

Contreras, Eguía y Solano (20 I I) realizaron un acercamiento al uso educativo que permiten los videojuegos en línea, al tratar de explicar cómo una herramienta de ocio como Monturiol el joc puede llegar a ser útil para un fin educativo. Estos autores recalcaban que el valor que obtiene el videojuego es complementario a la enseñanza que se recibe en las aulas, y no sustituye a la enseñanza que un profesor puede proporcionar, siendo la meta en todo caso, intentar que el estudiante relacione contenidos educativos adquiridos anteriormente, con contenidos que un videojuego puede proporcionarle, y destacando la importancia del profesor para que sea un guía a través del juego y empleen los videojuegos de forma controlada y responsable, ya que el juego aplicado en el aula debe ser una actividad conducida por el docente y por tanto, con una finalidad determinada, como un fin educativo.

García Blanco (20 I3) realizó durante el curso 20 I I-20 I2, un estudio para valorar algunos aspectos sobre los videojuegos y su utilización en el aula, comprobando que en opinión del alumnado, los videojuegos pueden ser educativos, aunque conocen pocos y los utilizan menos aún. Por ello, propone la creación y desarrollo de un videojuego, un mundo virtual, un metarverso donde los alumnos puedan realizar, por ejemplo, pirámides sin arena utilizando para ello cálculos matemáticos, trigonométricos y geométricos, historia, geografía y localización en un mapa, relieve del terreno, formas de negociación, compra-venta de materiales, etc.; generando un gran abanico de posibilidades ya que un mundo virtual tiene un objetivo educativo donde de forma transversal se pueden completar las distintas áreas de los currícula, pueden estar trabajándose las competencias básicas descritas en la normativa (LOE, 2006), todo ello desde la inclusión de todos los alumnos en el aula y promocionando la interacción en grupos colaborativos, ya que se pueden generar distintos perfiles por los cuales los alumnos han de ir pasando, o realizar distintas tareas para conseguir un único objetivo.

\section{Conclusiones y prospectiva investigadora}

A pesar de haber encontrado múltiples investigaciones acerca de la utilización de los videojuegos en el aula, son pocas las experiencias de uso de estas plataformas de ocio interactivas. 
El profesorado sigue resistiéndose a integrar estos dispositivos, creados normalmente para divertir y entretener, pensando en que pueden suponer más una distracción que un beneficio educativo.

Sin embargo, la mayoría de estos estudios muestran las posibilidades didácticas que los videojuegos poseen, exponiendo las grandes ventajas que estas herramientas tienen en el desarrollo de distintas competencias tales como "aprender a aprender" o "trabajo cooperativo".

Además, el desarrollo de la creatividad en el alumnado es una de los aspectos positivos que encontramos en diversos estudios, bien sea con la realización de cortometrajes, la creación de tus propios juegos interactivos digitales o el diseño de historias o paisajes.

La propuesta que estamos llevando a cabo en el Centro de profesorado SAFA-Úbeda con el alumnado del Grado de Educación Infantil es trabajar los videojuegos en el aula desde dos vertientes: educar con los videojuegos y educar por los videojuegos, es decir, por un lado estamos utilizando videojuegos comerciales para trabajar educación en valores, para lo cual se seleccionan videojuegos de diferentes géneros para extraer valores y contravalores y así fomentar el pensamiento crítico en el alumnado. Por otro lado, educamos por los videojuegos creando nuestros propios juegos educativos, bien utilizando el software que contienen algunos videojuegos comerciales, bien utilizando las herramientas que la web 2.0 y el multimedia ponen a nuestro alcance. En este sentido, la creación de contenidos y aplicaciones educativas para smartphones y tablets ha abierto un nuevo campo para crear juegos educativos de una forma sencilla.

\section{Bibliografía}

ADESE (Asociación Española de Distribuidores y Editores de software de entretenimiento) (20II). El videojugador español: perfil, hábitos e inquietudes de nuestros gamers. Recuperado el 30/10/2013 de http://www.adese.es/index. php?option $=$ com_content\&view $=$ article\&id $=10 \& \mid$ temid $=6$.

Aguiar Perera, M. V. y Farray Cuevas, J. I. (2003). Los Videojuegos. Comunicación y pedagogía, 191, 33-36.

Armando, J. (2010). Lo que podemos aprender de los videojuegos sobre la enseñanza y los materiales educativos. Revista Latinoamericana de Tecnología Educativa RELATEC, 9 (I), 29-4I. Recuperado el 25/10/20I3 de http://campusvirtual.unex.es/cala/editio/ 
Balaguer, R. (2002). Videojuegos, internet, infancia y adolescencia del nuevo milenio. Revista KAIROS, 6 (10). Recuperado el 10/10/2013 de http://www. cibersociedad.net/archivo/articulo.php?art $=6$.

Contreras, R. S., Eguía, J. L. y Solano, L. L. (20I I). Videojuegos como un entorno de aprendizaje. Icono I4, 9 (2), 249-26I.

Durall Gazulla, E. (2009). Mas allá del entretenimiento: Juegos Serios. Comunicación y Pedagogía, 239-240, 19-23.

Estallo, J. A. (1995). Los videojuegos. Juicios y prejuicios. Barcelona: Planeta.

Etxeberría Balerdi, F. (1998). Videojuegos y Educación. Comunicar: Revista Científica Iberoamericana de Comunicación y Educación, 10, I7I-180.

Farray, J. I., Aguiar, V., Bonny, A. y Calvo, M. L. (2002). Videojuegos: instrumento de cultura vs cultura de la tortura. En J. I. Farray, Cultura y educación en la Sociedad de la información. A Coruña: Netbiblo. Recuperado el I3/I0/20I3 de http://balboa. unex.es/crai/personal/evte/josefal.doc.

Garaigordobil, M. (1990). El juego como instrumento de maduración del niño. En C. Maganto, (Coord.), Orientación e Intervención psicológica del niño en la familia (pp. 33-53). San Sebastián: Universidad del País Vasco. Cuadernos de Extensión Universitaria.

García Blanco, S. (2013). Construir pirámides sin arena, iutopía? Investigación sobre la opinión acerca de la utilización de los videojuegos en el aula. Creatividad y sociedad, 2 I, I-34.

Gifford, B.R. (199I). The learning society: Serious play. Chronicle of Higher Education, 7.

Gros Salvat, B. (2009). El uso de los videojuegos para la formación universitaria y corporativa. Comunicación y Pedagogía, 239-240, I4-I8.

Grupo F9 (2004). Acceder a la cultura informática a través de los videojuegos. Documento electrónico. Recuperado el I0/I2/09 de http://www.xtec.es/ abernat.

Huerta Rojas, F. (2003). Socialización genérica de adolescentes en la ritualidad de los juegos virtuales. Comunicación y pedagogía, 191, 73-78.

Huerta Rojas, F. (2005). La violencia virtual: una experiencia de los jóvenes en las videosalas. Estudios Sociales, 13 (26), I72-206. 
Levis, D. (1997). Los videojuegos, un fenómeno de masas. Barcelona: Paidós.

Llorca Díez, M.A. (2009). Hábitos y uso de los videojuegos en la comunicación visual: influencia en la inteligencia espacial y en el rendimiento escolar. Tesis Doctoral inédita. Universidad de Granada.

Maldonado, A. (1999). Nuevos fenómenos lúdicos en la adolescencia. En actas del VI Congreso Nacional de Ludotecas (p. 98). Valencia: AlJU.

Marín Díaz, V. (2013). Los videojuegos y los juegos digitales como materiales educativos. Posibilidades educativas de los videojuegos y juegos digitales en educación inclusiva. Madrid: Síntesis.

Marquès Graells, P. (2000). Videojuegos. Las claves del éxito. Cuadernos de Pedagogía, 29I, 55-62.

Morales, E. (2009). El uso de los videojuegos como recurso de aprendizaje en educación primaria y Teoría de la Comunicación. Diálogos de la Comunicación, 78.

Pascual Sevillano, M. A. y Ortega Carrillo, J. A. (2007). Videojuegos y Educación. En Ortega Carrillo, J.A. y Chacón Medina, A. (Coords.), Nuevas Tecnologías para la Educación en la era digital (pp. 207-228). Madrid: Pirámide.

Pérez García, A. (2010). La integración curricular del cine digital en la formación inicial de los maestros: perspectivas didácticas y creativas. Tesis Doctoral inédita. Departamento de Didáctica y Organización Escolar. Universidad de Granada.

Pérez García, A. y Ortega Carrillo, J. A. (20I I). El potencial didáctico de los videojuegos: The Movies, un videojuego que fomenta la creatividad audiovisual. Revista etic@net, 10, Recuperado de: http://www.ugr.es/ sevimeco/revistaeticanet/ numerol0/Articulos/Formato/articulo2.pdf

Pérez Martín, J. (2008). Penetración de los videojuegos educativos e infantiles en España desde el 2005 al 2007. Comunicación y Pedagogía: Nuevas tecnologías y recursos didácticos, 229, 23-28.

Ortega Carrillo, J. A. (2002). Análisis crítico de los valores que transmiten los videojuegos: Descubriendo su potencial seductor de naturaleza subliminal. Recuperado el 9//0/2013 de http://www.ugr.es/si/txt/es/servinfo.htm.

Sádaba, R. y Naval, C. (2008). Una aproximación a la virtualidad educativa de los videojuegos. Teoría de la Educación: Educación y Cultura en la Sociedad de la Información, 9 (3), 167-183 Recuperado el I5/I0/2013 de http://www.usal.es/ teoriaeducacion/ 
rev_numero_09_03/n9_03_sadaba_naval.pdf

Torrico Franco, M. A. (2008). La Nintendo DS en el aula ordinaria. Propuesta metodológica. Comunicación y Pedagogía, 23I-232, 27-30.

Zagalo, N. (20l0). Alfabetización creativa en los videojuegos: comunicación interactiva y alfabetización cinematográfica. Comunicar, 35 (XVIII), 6I-68. 